\title{
MANIFESTATIONS ORL REVELATRICES DE LEUCEMIE AIGUE A PROPOS DETROIS OBSERVATIONS
}

\author{
S. TRABELSI, S. TABABI, L. OUERTATANI, I. BEN GHORBEL*, S. KHARRAT, N. BEN RHOMDHANE **, \\ N. BELTAIF, S. SAHTOUT, G. BESBES, M.H. HOUMAN*, S. HACHICHA. \\ SERVICE D'ORL, HÔPITAL LA RABTA, TUNIS. \\ * SERVICE DE MÉDECINE INTERNE, HÔPITAL LA RABTA, TUNIS. \\ ** SERVICE D'HÉMATOLOGIE. HÔPITAL LA RABTA, TUNIS.
}

\begin{abstract}
Les manifestations oto-rhino-laryngologiques sont fréquentes au cours des hémopathies malignes et peuvent les révéler malgré qu'elles soient rarement évocatrices par leur seul aspect clinique car polymorphe non spécifique. Le bilan et les traitements sont en règle assurés par les hématologistes Nous rapportons trois observations de leucémie aiguë (LA) révélée par des manifestations ORL.

MOTS CLES : Leucemie aiguë, manifestations ORL
\end{abstract}

\section{SUMMARY}

ENT expressions of blood diseases are not rare, and they can point to some of these diseases despite there are not specific. The haematologists ensure the diagnosis and provide treatment. We reported three cases of acute leukaemia revealed by ENT expressions.

KEY WORDS : Leukaemia - ENT manifestations

\section{INTRODUCTION}

Les manifestations oto-rhino-laryngologiques (ORL) sont fréquentes au cours des hémopathies malignes. Révélatrices ou non de la maladie, elles sont rarement évocatrices par leur seul aspect clinique car non spécifique. Un bilan hématologique simple, orienté par l'examen clinique, de même que des examens anatomopathologiques permettent d'en préciser l'étiologie ou de les rattacher à l'hémopathie. Nous rapportons trois observations de leucémie aiguë (LA) révélée par des manifestations . ORL.

\section{OBSERVATION}

Observation 1

Une femme âgée de 43 ans, a été hospitalisée en urgence pour une exploration étiologique d'adénopathie rétroangulo-maxillaire évoluant dans un contexte fébrile depuis 15 jours. L'examen clinique a révélé une fièvre à $39^{\circ} \mathrm{C}$, une pâleur cutanéo-muqueuse et des adénopathies rétroangulo-maxillaires fermes, sensibles et mobiles sans signes inflammatoires en regard. L'examen du cavum était normal. II existait une adénopathie inguinale droite de $2 \mathrm{~cm}$.

L'échographie cervicale a mis en évidence un amas d'adénopathies bilatérales, ovalaires hypo-échogénes d'aspect nécrosé et dont la plus grosse mesurait $3 \mathrm{~cm}$ de grand axe. La numération formule sanguine a objectivé une hyperleucocytose à 70000 éléments $/ \mathrm{mm3}$, une anémie à $7 \mathrm{~g} / \mathrm{dl}$ et une thrombopénie à $110 \quad 000$ éléments $/ \mathrm{mm} 3$. Le taux de prothrombine était à $48 \%$. Le myélogramme a mis en évidence une infiltration massive par des blastes. Le diagnostic d'une leucémie aiguë myéloïde (LAM) type 5 a été retenu. Une chimiothérapie à base de cytarabine et d'idarubicine associée à une antibiothérapie a été prescrite. L'évolution a été marquée par la survenue d'un épisode de neutropénie fébrile, compliquée d'un choc septique et d'une cellulite périnéale. Les hémocultures ont isolé un Pseudomonas. La patiente est décédée à $\mathrm{J} 13$ de chimiothérapie.

\section{Observation 2}

Un jeune de 16 ans a été hospitalisé pour exploration étiologique d'une dysphagie douloureuse évoluant depuis 3 semaines, associée à une altération de l'état général et à une fièvre non chiffrée. L'examen clinique a révélé la présence d'une hypertrophie amygdalienne unilatérale droite avec une hypertrophie gingivale associée à des adénopathies cervicales fermes indolores mobiles par rapport aux plans sous-jacents. Il existait une pâleur cutanéomuqueuse et une fièvre à $38,5^{\circ} \mathrm{C}$. La numération formule sanguine a révélé une pancytopénie avec une leuconeutropénie, une anémie à $6 \mathrm{~g} / \mathrm{dl}$ et une thrombopénie à 76000 éléments $/ \mathrm{mm} 3$. La vitesse de sédimentation était à $120 \mathrm{~mm}$ à la 1ère heure. Le myélogramme a mis en évidence un 
envahissement massif par des blastes. Le diagnostic d'une LAM type 5 a été retenu. Un traitement à base de cytarabine et d'idarubicine a été institué. L'évolution a été marquée par une rechute précoce fatale.

\section{Observation 3}

Une femme âgée de 23 ans, a été hospitalisée pour une exploration étiologique d'une hypertrophie parotidienne bilatérale et des deux glandes sous maxillaires évoluant depuis 2 mois avec amaigrissement chiffré à 5 kilogrammes. L'examen clinique a objectivé une hypertrophie dure et sensible des glandes salivaires (parotides et sous maxillaires). Les amygdales étaient hypertrophiées et cryptiques.

L'échographie cervicale a révélé deux glandes sous maxillaires hypertrophiées, globuleuses, mesurant $45 \mathrm{~mm}$ de grand axe, d'échostructure hypoéchogéne hétérogène et hypervascularisées au Doppler. Les glandes parotides mesuraient $5 \mathrm{~cm}$ de grand axe chacune, renfermant plusieurs nodules hyper échogènes variant entre 5 et $10 \mathrm{~mm}$ de taille.

La numération formule sanguine a montré une hyperleucocytose à 48000 éléments $/ \mathrm{mm} 3$, une anémie macrocytaire à $9,9 \mathrm{~g} / \mathrm{dl}$ et une thrombopénie à 100000 éléments/mm3. La vitesse de sédimentation était à $120 \mathrm{~mm}$ à la première heure. II existait une hyper gamma globulinémie d'allure polyclonale à $<24 \mathrm{~g} / \mathrm{l}$ et une hyperuricémie à $80 \mathrm{mg} / \mathrm{l}$.

La radiographie thoracique et l'échographie abdominale étaient normales. Le myélogramme a objectivé une infiltration médullaire par des blastes. Le diagnostic d'une LAM a été retenu. Une chimiothérapie à base de cytarabine et d'idarubicine associée à une antibiothérapie a été prescrite. L'évolution a été rapidement défavorable avec survenue d'un choc septique.

\section{DISCUSSION}

Les LA sont des hémopathies malignes caractérisées par une prolifération monocionale à point de départ médullaire de cellules immatures myéloïdes ou lymphoïdes.

La leucémie aiguë lymphoïde est la plus fréquente des pathologies tumorales de l'enfant estimée à $25 \%$ de tous les cancers et à $75 \%$ de toutes les leucémies. Chez l'adulte, la fréquence de la LAM augmente exponentiellement au-delà de 40 ans (1).

Les manifestations cliniques sont très variables. Certains patients vont présenter des infections ou des hémorragies au moment du diagnostic alors que pour d'autres malades, la maladie sera asymptomatique, diagnostiquée au cours d'un examen physique ou d'un bilan sanguin de routine $(1,2,3)$.

La symptomatologie clinique est en rapport soit avec la prolifération tumorale, 'soit avec l'inhibition de l'hématopoïèse normale (3).

Cette hémopathie va se manifester par une pâleur, une léthargie, un malaise, un saignement et/ou une fièvre. L'infiltration massive du foie, de la rate et des ganglions est classique et de mauvais pronostic $(1,3)$.

Les manifestations ORL en rapport avec une prolifération tumorale peuvent être soit des adénopathies cervicales comme c'était le cas de nos trois patients, soit une infiltration du tissu amygdalien et/ou une hypertrophie gingivale (4) comme dans notre observation 2 . L'infiltration des glandes parotides et sous maxillaires (5), comme dans notre observation 3, a été également rapportée, alors que la paralysie faciale par infiltration des glandes parotides n'a été décrite qu'une seule fois (6).

L'infiltration leucémique de l'oreille moyenne et de la mastoïde ont été également décrits $(7,8)$. L'atteinte des nerts crâniens témoigne de l'infiltration méningée, siège classique d'une rechute de la leucémie $(3,9)$.

L'infiltration de l'os temporal est bien documentée dans la littérature, et peut se présenter sous différentes formes à type de lésions de l'oreille externe, épanchement séreux de l'oreille moyenne, une surdité de transmission ou de perception, mastoïdite aiguë ou de paralysie faciale $(7,8,9)$.

D'autre part, les manifestations liées à l'inhibition de l'hématopoïèse normale sont les complications infectieuses et les accidents hémorragiques. Les éléments orientant vers le diagnostic sont la pâleur, un purpura siégeant au niveau des muqueuses buccopharyngées, une hémorragie de la muqueuse labiale associée à des aphtes et des ulcérations secondaires à la neutropénie $(1,3)$.

Le diagnostic de LA repose sur les données de l'hémogramme et du myélogramme. L'association d'une anémie normocytaire normochrome arégénerative, d'une neutropénie avec un taux de lymphocytes normal et myélemie, d'une thrombopénie et la présence de cellules anormales blastiques est caractéristique. Le myélogramme permet alors de confirmer le diagnostic en révélant une infiltration par plus de $30 \%$ de blastes (3).

En absence de traitement l'évolution est rapidement fatale souvent dans les mois qui suivent le diagnostic. Le traitement de la LA sera double reposant sur une chimiothérapie et une radiothérapie associée à une greffe de cellules souches $(1,3)$.

\section{CONCLUSION}

Les manifestations ORL au cours des leucémies sont polymorphes et non spécifiques. Elles peuvent être inaugurales. Le diagnostic est orienté par des arguments épidémiologiques et cliniques mais l'examen anatomopathologique et le bilan hématologique restent décisifs. 


\section{REFERENCES}

1-Brette MD, Monteil JP. Manifestations oto-rhino-laryngologiques des hémopathies de l'adulte. Encyclopédie Médico-Chirurgicale 20-915-A-10.

2-Bais A, Uppal K, Logani KB, Narayan S. Acute leukaemic cell infiltration of the nose. J Laryngol Otol $1992 ; 106: 261-3$

3-Najman A, Verdy E, Potron G, Isnard F. Hématologie. Paris :Ellipses, 1994. 4-Hou GI, Tsai ML. Primary gingival enlargement as a diagnostic indicator in acute myelomonocytic leukaemia. A case report. J Periodentol 1988; 59:244-8. 5-Fukuzawa J, Akaishi T, Tanaka H, Nakanishi K, Okada M, Haneda T, Kikuchi $K$. Adult T-cell leukemia with invasion by malignant cells of the parotid glands diagnosed by Ga-67 imaging. Clin Nucl Med 1996 ; 21(12):964-6.

6-Rhee D, Myssiorek D, Zahtz G, Diamond A, Paley C, Shende A. Recurrent attacks of facial nerve palsy as the presenting sign of leukemic relapse. Laryngoscope 2002; 112(2):235-7.
7-Paparella MM, Berlinger NT, Oda M, el-Fiky F. Otological manifestations of leukemia. Laryngoscope 1973; 83(9):1510-26.

8-Zechner G, Altmann F. The temporal bone in leukemia. Histological studies. Ann Otol Rhinol Laryngol 1969; 78(2):375-87.

9-Levy R, Har-El G, Segal K, Sidi J. Acute myelogenous leukemia presenting as facial nerve palsy. A case report. Int J Pediatr Otorhinolaryngol 1986 ; 12(1):49. 53. 